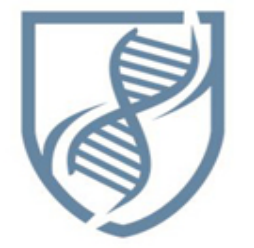

Journal of Bioscience and Applied Research

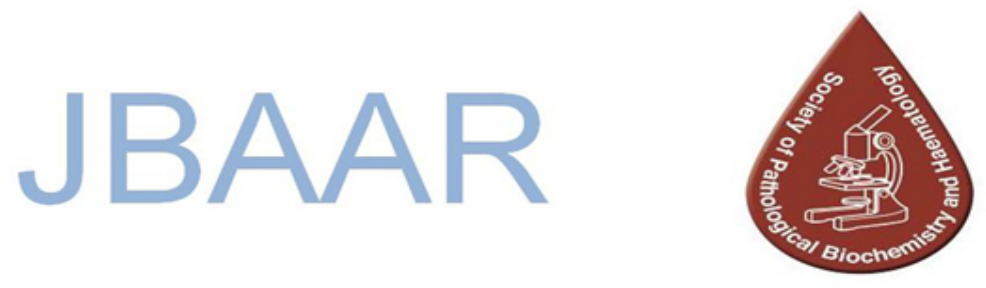

WWW.JBAAR.ORG

\title{
Effect of calcium ionophore A23187 on the sperm in intracytoplasmic sperm injection
}

\author{
Abd El-Wahab El-Ghareeb ${ }^{1}$, Ibrahim Yahia Abd el-Kadr ${ }^{1}$, Amr Tharwat Al Ahwany ${ }^{2}$, \\ Aziza Mansour $\mathrm{Ali}^{3}$, Ola Ismail Hassan ${ }^{3}$ \\ 1- Zoology Department, Faculty of Science, Cairo University, Egypt. \\ 2- Andrology Department, Faculty of Medicine, Cairo University, Egypt. \\ 3- Obstetrics \& gynecology department, International Islamic center for \\ population studies and research, Al-Azhar University. \\ (Corresponding author email:drelghareeb@yahoo.com))
}

\begin{abstract}
The present study evaluated the effect of calcium ionophore A23187 on sperm in intracytoplasmic sperm injection (ICSI) cycles using sperm from different sources. The 80 cycles evaluated were divided into four subgroups according to sperm quality and origin (normospermiaoligoathenoteratospermia-epidermal-testicular), subgroups were further split into experimental groups, depending on whether the spem treated or not treated with calcium ionophore. For each experimental subgroup, ICSI outcomes were compared between groups. In conclusion we found that using calcium ionophore increase the fertilization rate in case of sperm treatment with calcium ionophore before ICSI for oligoathenoteratospermia (OAT) \& testicular subgroups, with enhancing embryos quality in testicular subgroup only .
\end{abstract}

Keywords: Calcium ionophore A23187, fertilization rate, embryos quality, ICSI

\section{Introduction}

Intracytoplasmic sperm injection (ICSI) is a method of micromanipulation used to deposit a sperm directly into oocyte cytoplasm. When all other forms of assisted fertilization are unsuccessful, ICSI is the method of choice to overcome male infertility. ICSI has enabled fertilization of oocytes from patients whose partners have extremely low numbers of viable sperm and a very low probability of achieving fertilization after conventional spermoocyte incubation in vitro. ICSI is possible with sperm obtained from ejaculate, percutaneous epididymal sperm extraction, or testicular sperm extraction (Codreanu et al., 2013).

For all ages and with all different sperm types, the fertilization rate after ICSI is reported to be approximately $70-80 \%$. Although the fertilization rates after ICSI are relatively high, some injected oocytes fail to become fertilized despite the presence of a sperm in the cytoplasm and the absence of immaturity or degenerative alterations (Codreanu et al., 2013).

The terminology "failed fertilization" typically refers to failure of all the available mature MII oocytes to be fertilized. The principal cause of failed fertilization has been attributed to an oocyte activation disruption whether related to female or male gamete dysfunction (Neri et al., 2014). The Incidence of total fertilization failure (TFF) after ICSI is 1-5 \% (Koyono et al., 2012).

Fertilization failure after ICSI may occur because of the following reasons. First, the injected oocyte may fail to initiate the biochemical processes necessary for oocyte activation (Codreanu et al., 2013). Second, the biochemical processes are initiated, but they may not occur normally, thus leading to incomplete activation. Third, the sperm may remain poorly accessible to oocyte factors required for chromatin decondensation and formation of the male pronucleus (Mansour et al., 2009). Both sperm and oocyte factors are believed to be involved in failed oocyte activation after ICSI (Eftekhar et al., 2012). 
Oocyte activation results in the release of the oocyte from its metaphase II (MII) arrested state and leads to further embryo development (Vanden Meerschaut et al., 2013).

Failure of sperm decondensation has been related to sperm quality, high sperm DNA fragmentation and inadequate calcium activation (Nasr-Esfahani et al., 2010). In 1951 Chang, while studying rabbits, and Austin, while working on rats, each independently reported that mammalian sperm must reside in the female reproductive tract for a finite period of time before they gain the ability to fertilize ova. One year later, Austin introduced the term "capacitation" when he stated that "the sperm must undergo some form of physiologic change or capacitation before it is capable of penetrating the egg. A Capacitation is now commonly regarded as the reversible, prefertilization activation process of sperm (Brannigan and Lipshultz, 2008).

Sperm capacitation is a multistep process that involves modifications to plasma membrane lipid composition, increase of permeability to ions, acquisition of hyperactivated motility and an increase in the phosphotyrosine content of specific sperm proteins (Zhang et al., 2010). Fraser, 1998 reported that capacitation is a comparatively slow event that requires several hours to complete and is mainly regulated by a modest rise in $\left[\mathrm{Ca}^{2+}\right]_{\mathrm{i}}$, whereas the acrosome reaction is an exocytosis process that occurs very rapidly (within a minute) and is triggered by a large influx of $\left[\mathrm{Ca}^{2+}\right]_{\mathrm{i}}$ (Michaut,2000).

The primary source of $\mathrm{Ca}^{2+}$ for spermatozoa is the external environment: the fallopian tube in the female reproductive tract (in vivo) and culture media (in vitro) (Foresta and Rossato, 1997), and simultaneously increasing $\left[\mathrm{Ca}^{2+}\right]_{\mathrm{i}}$ regulates the release of $\mathrm{Ca}^{2+}$ into the cell. Therefore, how $\mathrm{Ca}^{2+}$ crosses into cells through the sperm plasma membrane is a matter of paramount importance (Pietrobon et al., 1990). Measuring cytoplasmic $\mathrm{Ca}^{2+}$ levels by using the fluorescent $\mathrm{Ca}^{2+}$ indicator indo-1 proved that spermatozoa hyperactivation is potentially regulated by $\mathrm{Ca}^{2+}$ influx (Rahman et al., 2014).

Incubation of spermatozoa with an extracellular $\mathrm{Ca}^{2+}$ source induces hyperactivation in mammalian spermatozoa (Marquez and Suarez, 2008).

To improve ICSI efficacy, appropriate sperm and oocyte activation prior to or after ICSI is necessary to achieve normal fertilization/early embryo development. (Tasripoo et al., 2012).

A23187 is a divalent cation ionophore obtained from a strain of Streptomyces chartreusis (Chaney et al., 1974).

Ionophores are lipid-soluble molecules that complex cations and transport them across a variety of membranes (Reed, 1972). Since Calcium Ionophore A23187 is highly selective for $\mathrm{Ca}^{2+}$ it is commonly used to increase intracellular $\mathrm{Ca}^{2+}$ levels in intact cells (Wang et al., 1994).

In this study, we evaluated the effect of calcium ionophore A23187 on sperms in increasing the fertilization rate in ICSI, with the assessment of the embryos quality.

\section{Materials and Methods}

\section{Experimental design}

- This study involved 80 ICSI patients, underwent the ICSI program at the International Islamic Center for Population Studies and Research, AlAzhar University, Cairo, Egypt, between January to September 2014.

- The age of women included in this study is under 38 years at the start of the treatment. Extensive counseling was given beforehand to the couples and the Internal Ethical Committee approved the study.

For the 80 ICSI cycle were divided into four experimental subgroups according to sperm origin and type, 20 ICSI cycles for each subgroup:

1.Normospermia subgroup (ejaculated).

2.Oligoathenoteratospermia (OAT) subgroup (ejaculated).

3.Epididymal subgroup (PESA).

4.Testicular subgroup (TESE).

For each experimental subgroup, calcium ionophore A23187 treated group were compared to control group (non-treated with calcium ionophore A23187.

\section{Ovarian Stimulation}

Women received ovarian stimulating drugs according to the ART protocols. Follicular development was monitored by ultra sound scanning and serum estradiol and progesterone levels. Patients received 10,000 IU of Human Chronic Gonodotrophin (HCG) when most of the follicles measured more than $18-20 \mathrm{~mm}$ in diameter.

\section{Sperm Preparation}

Sperm obtained from ejaculate, percutaneous epididymal sperm extraction, or testicular sperm extraction.

The normospermia patient who follow the WHO 2010 parameters.Oligoathenoteratospermia patients who have semen parameters lower than the WHO semen count, motility and morphology parameters.

Sperm preparation done by a discontinuous density gradient centrifugation method using Isolate sperm separation media (Irvine Scientific, Santa Ana, CA, USA) (Makkar et al., 2001). The bottom fraction was aspirated and washed twice with Sydney IVF Gamete Buffer (Cook Sydney IVF Limited, National Technology Park, Ireland, UK) at 1200 r.p.m. for $8 \mathrm{~min}$. The resulting sperm pellet after washing overlaid with the same medium to give a final volume of $0.3-0.5 \mathrm{ml}$.

Epididymal spermatozoa obtained by PESA, is performed under local anaesthesia using a 27-gauge needle that inserted into the epididymis. Gentle, negative pressure is applied as the epididymal fluid was aspirated.

Testicular spermatozoa were obtained via TESE, after administration of cord block anaesthesia. Samples extracted from the testis were dissected with small scissors and checked for the presence of spermatozoon.

PESA and TESE Samples were collected in a Falcon tube and washed by centrifugation at (1800 r.p.m for 10 
min) with minimum culture medium. The pellet is suspended in 0.3-0.5 ml Sydney IVF Gamete Buffer (Cook Sydney IVF Limited, National Technology Park, Ireland, UK).

\section{Calcium ionophore A23187 preparation:}

Calcium ionophore A23187 (Calcium ionophore A23187(C7522) Sigma Free Acid; Sigma Chemical Co., St Louis, MO, USA) was dissolved in $1 \mathrm{ml}$ of cell culturetested dimethylsulphoxide (DMSO (D2650); SigmaAldrich Chemie, Belgium), to get the concentration of one mmol/l of Calcium ionophore A23187 stock solution.

The final solution containing $5 \mu \mathrm{mol} / 1$ ionophore was prepared by adding $5 \mu \mathrm{ml}$ from calcium ionophore stock solution to $995 \mu \mathrm{ml}$ of Cook Cleavage medium (Cook Sydney IVF Limited, National Technology Park, Ireland, UK) just before ICSI.

Sperm treatment by calcium ionophore A23187.

Sperms from different sources and quality (normospermia- oligoathenoteratospermia - epidermal testicular) were preparation and afterwards the prepared samples were aliquoted into two parts. The first is the control. For the second aliquot, we adjust the volume to be $0.495 \mu \mathrm{l}$. For sperm activation we add $5 \mu \mathrm{ml}$ stock solution of A23187 (Sigma- Chemical Co., St Louis, MO, USA) in dimethyl sulphoxide (DMSO; Sigma) to make a final concentration of $10 \mu \mathrm{mol} / \mathrm{l}$ A23187. Sperm is incubated with the calcium ionophore for 1 hour before ICSI.

\section{Oocyte Aspiration}

Under general anaesthesia, gynaecologist aspirates the oocytes by using a specialized, ultrasound-guided needle (Labotect aspiration catheter, Germany) at $36 \mathrm{~h}$ after HCG injection. Warmed HEPES buffered medium (Irvine Scientific, Irvine, CA, USA) was used for handling and washing of oocytes. Oocyte-cumulus were identified by the embryologist, then washed and incubated in Sydney IVF Fertilization Medium (Cook Sydney IVF Limited, National Technology Park, Ireland, UK), at $37^{\circ} \mathrm{C}$ and $6 \% \mathrm{CO} 2$ for approximately 1 hour.

\section{Oocytes Denudation}

Oocytes were placed in a $100 \mu 1$ drop of buffer containing 80 IU hyaluronidase/ml (Irvine Scientific, Irvine, CA, USA) for 30seconds, and then the oocyte was removed and placed in $100 \mu \mathrm{l}$ drop of Sydney IVF Gamete Buffer (Cook Sydney IVF Limited, National Technology Park, Ireland, UK). The corona cells were removed by gentle aspiration of the oocyte in and out by a sterile flame polished Pasteur pipette. When stripping was completed, the oocytes washed in Sydney IVF Gamete Buffer (Cook Sydney IVF Limited, National Technology Park, Ireland, UK). All manipulations are carried on a heated microscope stage.

\section{Oocytes Injection Procedure}

The ICSI procedure is carried out in a plastic microinjection dish, tissue-culture-grade (353004, Petri Dishes (BD Falcon)), containing micro droplets Sydney IVF Gamete Buffer medium (COOK, COOK IRELAND LTD, AUSTRALIA), covered with $4 \mathrm{ml}$ of sterile equilibrated mineral oil (Irvine Scientific, Irvine, CA, USA). A fraction ( $1 \mu$ ) of the sperm suspension is added to the periphery of the central PVP 10\%; polyvinylpyrrolidone (Irvine Scientific, Irvine, CA, USA) droplet, separate medium droplets are used in cases of epididymal or testicular sperm and the best selected sperm is picked up and placed into the droplet of PVP. Sperm injection was carried out $38 \mathrm{~h}$ after recombinant HCG trigger on the heated stage $\left(37^{\circ} \mathrm{C}\right)$ of an inverted microscope (Olympus 1x71) equipped with Hoffman modulation contrast optics, x4, x10 and x20 objective lenses and 10x eye pieces, with heated stage to maintain the temperature at $37{ }^{\circ} \mathrm{C}$, and automatic manipulators (Narashige, Japan). Both holding and injection pipettes were obtained commercially (Humagen Fertility Diagnostics, USA). The sperm cells scanned for the sperm selection. Immobilization of the sperm is done by hitting its tail with the injection micropipette, and then aspirated tail first into the pipette. The oocyte to be injected is attached to holding pipette at 9 o'clock using gentle suction, and then rotated such that the first polar body was located at either the 6 o'clock or 12 o'clock position.

The oocytes of each ICSI cycle will be divided into 2 parts, the first part injected with the control specimen. The second part injected with the sperm specimen which treated by calcium ionophore A23187.

After ICSI, oocytes are rinsed and separated, the oocytes transferred into a $20 \mu \mathrm{l}$ droplets Sydney IVF cleavage medium (Cook Sydney IVF Limited, National Technology Park, Ireland, UK)., for culture medium and incubated under mineral oil at $37^{\circ} \mathrm{C}$ and $6 \% \mathrm{CO} 2$ in order to follow their further development

After 18 hours from injection, the oocytes are evaluated for fertilization, and evaluated for the second time at the day of embryo transfer.

\section{Day 1 Scoring (Assessment of Fertilization)}

At about 18-20 h after ICSI, oocytes were examined for the presence of pronuclei and polar bodies by using an X20 objective lens on inverted microscope with Hoffman modulation contrast optics.Fertilization was considered normal when two clearly distinct pronuclei were present.

\section{Assessing Cleavage-Stage Embryos (Days 2 and 3)}

Embryo quality was evaluated on inverted microscope with Hoffman modulation contrast optics with X20 objective lens, and the following parameters were be in consideration : (i) the number of blastomeres; (ii) the fragmentation percentage; (iii) variation in blastomere symmetry; (iv) the presence of multinucleation; and (v) defects in the zona pellucida and cytoplasm.

Day 3 embryos classified as described previously from grade 1 to 6 , grade 1 and 2 represents the embryos with good quality.

\section{Statistical analysis}

The data from all of the experiments described above were tested for statistical significance either by analysis of variance, ANOVA or Duncan's multiple range tests to determine difference in means using Statistical Analyses Systems. Results were considered to be significant at the 5\% critical level ( $\mathrm{P}<0.05)$. (SAS, 2000). 


\section{Results}

80 ICSI cycles involved in this study, 20 ICSI cycle in each subgroup. Total of 583 oocytes injected in this group, 415 oocytes were fertilized, with fertilization rate is $71.1 \%$. (table 1, Figs.1\&2)).

For the first subgroup normospermia:

- Total of 153 oocytes were injected, from them 142 oocytes were fertilized with fertilization rate of $92.8 \%$.

- 76 oocytes were injected with sperms treated with calcium ionophore A23187; from them 71 oocytes were fertilized with fertilization rate of $93.4 \%$.

- 77 oocytes were injected with non-treated sperms (control group); from them 71 oocytes were fertilized with fertilization rate of $92.2 \%$.

- $\quad$ So the fertilization rate of oocytes injected with sperms treated with calcium ionophore A23187 is approximately as the fertilization rate of oocytes injected with non-treated sperms (control group), as $\mathrm{P}$ value is $0.7806(\mathrm{P}<0.05)$; which is non-significant value.

- Embryos with good quality obtained from oocytes fertilized with sperm treated with calcium ionophore A23187 were 52 from 71 embryos with percentage of 73.2\%.

- Embryos with good quality obtained from oocytes fertilized with nontreated sperm (control group) were 49 from 71 embryos with percentage of $69 \%$.

- $\quad$ So the percentage of embryos with good quality from the treated group and non-treated group (control group) is almost the same, as the $\mathrm{P}$ value is $0.2654(\mathrm{P}<0.05)$, non-significant value.

For the second subgroup oligoathenoteratospermia (OAT):

- $\quad$ Total of 139 oocytes were injected, from them 88 oocytes were fertilized, with fertilization rate of $63.3 \%$.

- $\quad 58$ oocytes were injected with sperms treated with calcium ionophore A23187; from them 42 oocytes were fertilized with fertilization rate of $72.4 \%$.

- $\quad 81$ oocytes were injected with non-treated sperms (control group); from them 46 oocytes were fertilized with fertilization rate of $56.8 \%$.

- $\quad$ So the fertilization rate of oocytes injected with sperms treated with calcium ionophore A23187 is more than the fertilization rate of oocytes injected with nontreated sperms (control group), as the $\mathrm{P}<0.0001$; as it is highly-significant value .

- $\quad$ Embryos with good quality obtained from oocytes fertilized with sperm treated with calcium ionophore A23187 were 19 from 42 embryos with percentage of $45.2 \%$.
- $\quad$ Embryos with good quality obtained from oocytes fertilized with nontreated sperms (control group) were 22 from 46 embryos with percentage of $47.8 \%$.

- $\quad$ So the percentage of embryos with good quality from the treated group and non-treated group (control group) is almost the same, as the $\mathrm{P}$ value is 0.3939 ( $\mathrm{P}<$ $0.05)$, non-significant value.

\section{For the third epididymal subgroup (PESA):}

- $\quad$ Total of 142 oocytes were injected, from them 101 oocytes were fertilized, with fertilization rate of $71.1 \%$.

- $\quad 58$ oocytes were injected with sperms treated with calcium ionophore A23187; from them 42 oocytes were fertilized with fertilization rate of $75.4 \%$.

- 53 oocytes were injected with non-treated sperms (control group); from them 40 oocytes were fertilized with fertilization rate of $68.5 \%$.

- $\quad$ So the fertilization rate of oocytse injected with sperms treated with calcium ionophore A23187 is approximately as the fertilization rate of oocytes injected with non-treated sperms (control group), as the $\mathrm{P}$ value is 0.0689 ( $\mathrm{P}<0.05$ ); as it is non-significant value .

- $\quad$ Embryos with good quality obtained from oocytes fertilized with sperms treated with calcium ionophore A23187 were 18 from 42 embryos with percentage of $42 \%$.

- $\quad$ Embryos with good quality obtained from oocytes fertilized with nontreated sperms (control group) were 27 from 61 embryos with percentage of $44.2 \%$.

- $\quad$ So the percentage of embryos with good quality from the treated group and non-treated group (control group) are almost the same, as the $\mathrm{P}$ value is 0.4537 ( $\mathrm{P}<$ $0.05)$, non-significant value.

\section{For the fourth the testicular subgroup (TESE):}

- $\quad$ Total of 149 oocytes were injected, from them 84 oocytes were fertilized, with fertilization rate of 56.3\%.

- $\quad 72$ oocytes were injected with sperm treated with calcium ionophore A23187; from them 46 oocytes were fertilized with fertilization rate of $63.8 \%$.

- $\quad 77$ oocytes were injected with non-treated sperm (control group); from them 38 oocytes were fertilized with fertilization rate of $49.3 \%$.

- $\quad$ So the fertilization rate of oocytes injected with sperm treated with calcium ionophore A23187 is more than the fertilization rate of oocytes injected with nontreated sperm (control group), as the $\mathrm{P}<0.0001$; and it is highly-significant value .

- $\quad$ Embryos with good quality obtained from oocytes fertilized with sperm treated with calcium ionophore 
- A23187 were 22 from 46 embryos with percentage of $47.8 \%$.

- $\quad$ Embryos with good quality obtained from oocytes fertilized with nontreated sperm (control group) were 15 from 38 embryos with percentage of
39.4\%. Therefore, the percentage of embryos with good quality from the treated group is more than percentage of embryos with good quality from nontreated group (control group), as the $\mathrm{P}$ value is $0.0044(\mathrm{P}<0.01)$, highlysignificant value.

Table(1) : The fertilization rate and percentage of embryos with good quality in the four subgroups normospermia subgroup, oligoathenoteratospermia (OAT) subgroup, epididymal subgroup (PESA), and the testicular subgroup (TESE), of the first group as oocytes injected with sperm treated with calcium ionophore A23187 (First Group).

\begin{tabular}{|c|c|c|c|c|c|c|c|c|c|c|}
\hline & & & \multicolumn{4}{|c|}{$\begin{array}{c}\text { Oocyte Injected with treated Sperm } \\
\text { by } \\
\text { Calcium Ionophore A23187 }\end{array}$} & \multicolumn{4}{|c|}{$\begin{array}{c}\text { Oocyte Injected with } \\
\text { Non- Treated Sperm by } \\
\text { Calcium Ionophore A23187 }\end{array}$} \\
\hline \multicolumn{3}{|c|}{$\begin{array}{c}\text { Sperm Sources } \\
\text { (Total Fertlized Oocyte No. /Total Oocyte No.)\% }\end{array}$} & \multicolumn{2}{|c|}{$\begin{array}{c}\text { Total } \\
\text { Fertilized } \\
\text { Oocyte No. / } \\
\text { Total } \\
\text { Oocyte No. }\end{array}$} & \multicolumn{2}{|c|}{$\begin{array}{l}\text { No. of } \\
\text { Good } \\
\text { Embryos } \\
\text { Quality }\end{array}$} & \multicolumn{2}{|c|}{$\begin{array}{c}\text { Total } \\
\text { Fertilized } \\
\text { Oocyte No. / } \\
\text { Total } \\
\text { Oocyte No. }\end{array}$} & \multicolumn{2}{|c|}{$\begin{array}{l}\text { No. of Good } \\
\text { Embryos } \\
\text { Quality }\end{array}$} \\
\hline Normospermia & $142 / 153$ & $92.8 \%$ & $71 / 76$ & $93.4 \%$ & $52 / 71$ & $73.2 \%$ & $71 / 77$ & $92.2 \%$ & 49/71 & $69.0 \%$ \\
\hline Oligoathenoteratospermia & $88 / 139$ & $63.3 \%$ & $42 / 58$ & $72.4 \%$ & $19 / 42$ & $45.2 \%$ & $46 / 81$ & $56.8 \%$ & $22 / 46$ & $47.8 \%$ \\
\hline PESA & $101 / 142$ & $71.1 \%$ & $40 / 53$ & $75.4 \%$ & $18 / 42$ & $42.0 \%$ & $61 / 89$ & $68.5 \%$ & $27 / 61$ & $44.2 \%$ \\
\hline TESE & $84 / 149$ & $56.3 \%$ & $46 / 72$ & $63.8 \%$ & $22 / 46$ & $47.8 \%$ & $38 / 77$ & $49.3 \%$ & $15 / 38$ & $39.4 \%$ \\
\hline
\end{tabular}

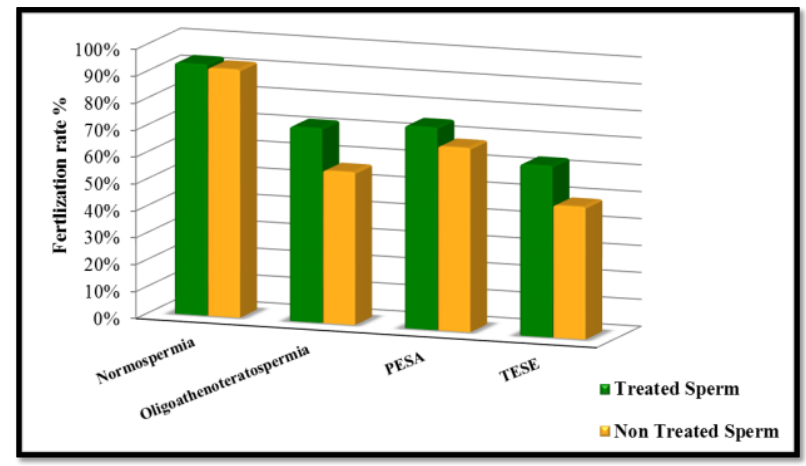

Figure (1): The fertilization rate of oocytes injected with sperms treated with calcium ionophore A23187 and the oocyte injected with non-treated sperm (control group), in the four subgroups (First Group). 


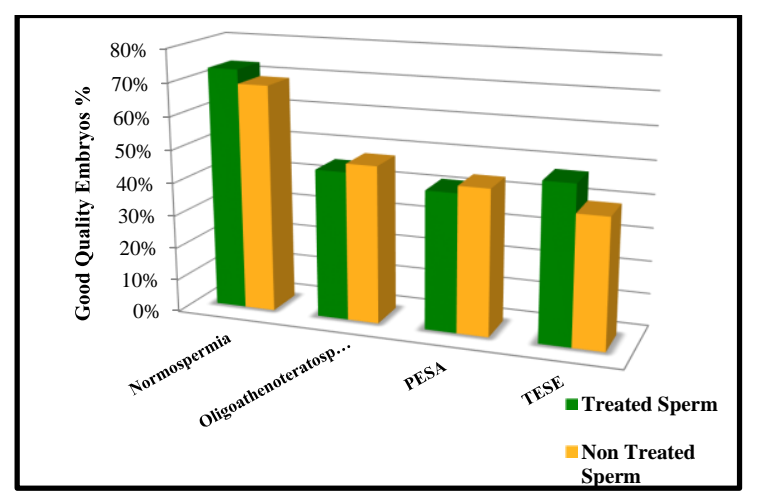

Figure (2): The percentage of embryos with good quality obtained from oocytes injected with sperms treated with calcium ionophore A23187 and the oocytes injected with non-treated sperms (control group), in the four subgroups (First Group).

\section{Discussion}

In this study, we tried to assess the effect of calcium ionophore A23187 on the sperm in enhancing the fertilization rate and embryos quality in ICSI cycles using sperm from different sources. The 80 cycles evaluated were divided into four subgroups according to sperm quality and origin normospermia,oligoathenoteratospermia, epidermal and testicular.

For the first subgroup normospermia, the calcium ionophore A23187 did not enhance the fertilization rate in the normospermia patients; it also did not improve embryos quality. For the second subgroup oligoathenoteratospermia (OAT), the calcium ionophore enhanced the fertilization rate in the oligoathenoteratospermia patients, but did not improve the embryos quality. For the third epididymal subgroup (PESA), the calcium ionophore A23187 did not enhance the fertilization rate in the epididymal subgroup (PESA); also did not improve quality of the embryos. For the fourth the testicular subgroup (TESE), the calcium ionophore enhanced the fertilization rate in the testicular subgroup (TESE) patients, it also enhanced the embryos quality.

In summary, we found that in ICSI cases when the sperm treated with $10 \mu \mathrm{l}$ calcium ionophore A23187 for 1 hour before ICSI, the fertilization rate of the oocytes in the four subgroup is more than the fertilization rate of the control subgroup but it is significant only in oligoathenoteratospermia (OAT) \& testicular subgroups. Also, the embryos quality, it is almost the same in all subgroups except in testicular subgroup the percentage of embryos with good morphology increased with significant value.

This study is somewhat in agreement with Liu and Baker (1998) study; they presented a study to determine the relationship between calcium ionophore A23187-induced acrosome reaction (AR) and sperm fertilizing ability in IVF patients. They found that calcium ionophore-induced AR is correlated with fertilization rates in vitro, although not strongly. While significant in patients with teratozoospermia, ionophore induced AR was not related to fertilization rate in patients with normal sperm morphology.

In ICSI, the first $\mathrm{Ca}^{2+}$ rise in the oocyte starts $20-30$ min after ICSI and originates from the oocyte cortex. This $\mathrm{Ca}^{2+}$ rise alone is insufficient to fully activate the oocyte .To sustain the oscillation function, sperm demembranization is necessary to facilitate the liberation of the cytosolic sperm factor responsible for the oscillator function (Heindryckx et al., 2005).

It is apparent that for oocyte activation to occur, the sperm plasma membrane has to disintegrate to let the perinuclear material blend within the ooplasm. Only when this happens, the sperm-activating factor is released and is subsequently able to target phosphatidylinositol 4, 5bisphosphate to cause an IP3 receptor mediated calcium release (Vanden Meerschaut et al., 2013).

Numerous signaling cascades chiefly regulate the fundamental sperm maturational process, and calcium $\left(\mathrm{Ca}^{2+}\right)$ plays a dynamic role in this process, as an intracellular second messenger. Several studies have hypothesized that elevation of sperm intracellular $\mathrm{Ca}^{2+}$ $\left(\left[\mathrm{Ca}^{2+}\right]_{\mathrm{I}}\right) / \mathrm{Ca}^{2+}$ influx regulates motility, hyperactivation, chemotaxis, capacitation, and the acrosome reaction and facilitates the spermatozoa reaching and fertilizing of an oocyte (Mannowetz et al.,2013). In vivo capacitation takes place in the female reproductive tract; however, it is also possible to capacitate spermatozoa in vitro by using particular media containing appropriate electrolytes and $\mathrm{pH}$ (Chang, 1951), as first demonstrated by Toyoda et al. (1971) in the mouse (Tateno et al., 2013).

In vitro the AR can be induced in capacitated spermatozoa by incubation with solubilized zona pellucida proteins, progesterone or calcium ionophore A23187. The ensuing signal transduction cascade invokes a host of enzymatic activities and an elevation in the cytosolic calcium concentration, subsequently leading to the fusion of the plasma and outer acrosomal membranes, which culminates in acrosomal exocytosis (Zhang et al., 2010). 
Capacitation is associated with significant alteration of the surface of the sperm, with various molecules being removed or rearranged (Brannigan et al., 2008).

The sperm plasma membrane is composed of a lipid bilayer interspersed with a number of proteins. Lipid types present include cholesterol, glycolipids, and phospholipids. The proteins found here can traverse the entire membrane from cytosolic compartment to extracellular space. These proteins have important functions, including activation of receptors and transport of ions (Brannigan et al., 2008).

Capacitation is characterized by a loss or reduction of cholesterol from the plasma membrane of spermatozoa. Membranes are a very dynamic collection of proteins and lipids that are capable of responding to various environmental signals that modify cellular activities (Brannigan et al., 2008).

Cholesterol has been shown to limit the insertion of proteins into lipid bilayers, to prohibit the movement of receptors in cell membranes and to change membrane protein conformation and thus alter their activity (Brannigan et al., 2008). The ratio of cholesterol to phospholipid, so important in the sperm membrane, controls fluidity and ion permeability in most biologic membranes, and the proportion of these two components change during capacitation (Brannigan et al., 2008). Various studies have shown in vitro that plasma membrane cholesterol content is reduced by $20 \%$ to $50 \%$, depending on the makeup of the capacitating medium. Collectively, these changes in sperm membrane composition are believed to be interrelated to subsequent changes in membrane ion transport and possibly membrane fusion (Brannigan et al., 2008).

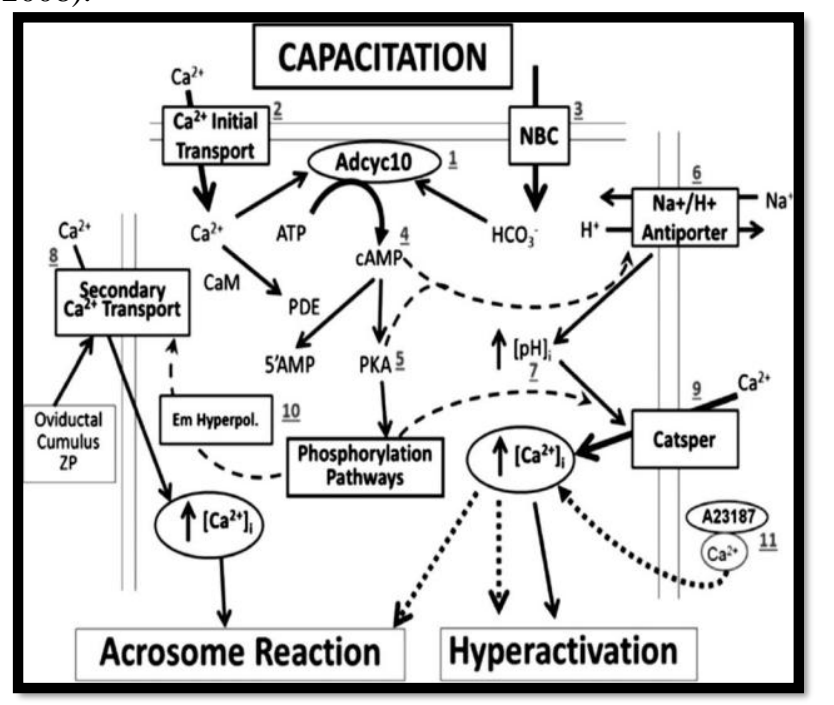

Figure (15): Working model of the sequence of events involved in preparation of spermatozoa for the acrosome reaction and hyperactivation under normal conditions and after $\mathrm{Ca}^{2+}$ ionophore treatment (Tateno et al., 2013).

Ionophores are lipid-soluble molecules that complex cations and transport them across a variety of membranes. Calcium ionophore A23187, used to transports extracellular $\mathrm{Ca}^{2+}$ into sperm. This ionophore increased respiration, motility and induces the acrosome reaction of sperm of a variety of animal species (Tateno et al., 2013).

When spermatozoa were treated with A23187, more than $95 \%$ of oocytes were fertilized. Furthermore, Ionophore-treated spermatozoa also fertilized $80 \%$ of oocytes, even in the absence of $\mathrm{HCO}_{3}^{-}$, a component essential for cAMP synthesis under normal in vitro conditions. Under these conditions, fertilized oocytes developed into normal offspring (Tateno et al., 2013).

Sperm treated with ionophore are able to fertilize without activation of the cAMP/PKA signaling pathway. Furthermore, they suggest that the cAMP/PKA pathway is upstream of an intracellular $\mathrm{Ca}^{2+}$ increase required for the acrosome reaction and hyperactivation of spermatozoa under normal in vitro conditions (Tateno et al., 2013).

Ionophore bypasses signaling events associated with sperm capacitations that are necessary for normal fertilization in vivo and in vitro (Tateno et al., 2013).

Figure (15) represents a working model of the sequence of events that occur during capacitation, acrosome reaction, and hyperactivation of spermatozoa under normal conditions (solid lines for known events, dashed lines for probable events). Figure (15) also suggests a way by which $\mathrm{Ca}^{2+}$ ionophore overcomes other signaling events and induces both the acrosome reaction and hyperactivation of spermatozoa (thin dotted arrows)(Tateno et al., 2013).

When fresh spermatozoa are placed in capacitationsupporting medium, the atypical adenylyl cyclase Adcy 10 (see (1) in Figure (15)) is quickly stimulated by the combined action of $\mathrm{Ca}^{2+}$ and $\mathrm{HCO}_{3}^{-}$, which enter spermatozoa through an initial $\mathrm{Ca}^{2+}$ transporter (2), and a $\mathrm{Na}^{+} / \mathrm{HCO}_{3}{ }^{-}$cotransporter (ㅁ) ), respectively (Demarco et al., 2003) (underlined numbers in parentheses cited in this paragraph refer to the underlined numbers in Figure (15)). Elevated cAMP (ㄴ) activates PKA (ㅁ) and the $\mathrm{Na}^{+} / \mathrm{H}^{+}$ antiporter (므), resulting in a $[\mathrm{pH}]_{\mathrm{i}}$ rise (므), which stimulates Catsper (ㅇ) . Some sperm components are phosphorylated during capacitation, include the secondary $\mathrm{Ca}^{2+}$ transporter (8) and the calcium channel protein Catsper (ㅁ) (Ren and Xia, 2010). The former, when stimulated by factors from the oviduct, cumulus, or zona pellucida, transports extracellular $\mathrm{Ca}^{2+}$ into the sperm head to induce the acrosome reaction. $\mathrm{Ca}^{2+}$ entering sperm flagellum through activated Catsper (9) induces hyperactivation. Alternatively, phosphorylation pathways could regulate $\mathrm{Ca}^{2+}$ channels by inducing hyperpolarization of the sperm plasma membrane potential (Em Hyperpol) (10) (Tateno et al., 2013).

Ionophore A23187 (11) raises the $\left[\mathrm{Ca}^{2+}\right]_{\mathrm{i}}$ of spermatozoa to induce both the acrosome reaction and hyperactivation, bypassing all ion transporters and cAMP/PKA pathways in spermatozoa (bold dotted arrows (Tateno et al., 2013).

In conclusion we found that using calcium ionophore increases the fertilization rate in case of sperm treatment with calcium ionophore before ICSI for oligoathenoteratospermia (OAT) \& testicular subgroups, with enhancement of embryos quality in testicular subgroup only. 


\section{References}

Brannigan, R, Lipshultz, L, (2008). Sperm Transport and Capacitation. Glob. libr. women's med., DOI 10.3843/GLOWM.1031

Chang MC. (1951). Fertilization capacity of spermatozoa deposited in fallopian tubes. Nature; 168: 697-8.

Chaney MO, Demarco PV, Jones ND, Occolowitz JL. (1974). The structure of A23187, a divalent cation ionophore. J. Am. Chem. Soc.; 96:1932-1933.

Codreanu D., Coricovac A. , Dracea L. M. L., Marinescu B. and Boleac I. (2013): Natural concepation following total fertilization failure with intracytoplasmic sperm injection in a couple with unexplained infertility: A case report.Rev. Med. Char. Soc.Med. Nat., Iasi.; 117(2). 431-8.

Demarco IA, et al. (2003). Involvement of a $\mathrm{Na}+$ /HCO-3 cotransporter in mouse sperm capacitation. J Biol Chem.; 278(9):7001-7009.

Eftekhar M, Mohammadian F, Yousefnejad F, Khani P, Aflatoonian A. (2012). Effect of calcium ionophore on unfertilized oocytes after ICSI cycles. Iran J Reprod Med 10(2): 83-86.

Foresta C, Rossato M. (1997): Calcium influx pathways in human spermatozoa. Molecular Human Reproduction. 3(1):1-4.

Heindryckx B, Van der Elst J, De Sutter P, Dhont M. (2005). Treatment option for sperm- or oocyte-related fertilization failure: assisted oocyte activation following diagnostic heterologous ICSI. Human Reproduction; 20:2237-2241.

Kyono K., Takisawa T., Nakajo Y., Doshida M. and Toya m. (2012). Birth And Follow Up Of Babies Born Following ICSI With Oocyte Activation Using Strontium Chloride or Calcium Ionophore A23187. Journal of Mammalian Ova Research ; 29:35- 40.

Liu DY., Baker HWG. (1998). Calcium ionophoreinduced acrosome reaction correlates with fertilization rates in vitro in patients with teratozoospermic semen. Human Reproduction; 13 (4):905-910.

Mansour R, Fahmy I, Tawab NA, Kamal A, ElDemery Y, Aboulghar M, et al. (2009). Electrical activation of oocytes after intracytoplasmic sperm injection: a controlled randomized study. Fertility and Sterility; 91:133-139.

Marquez B \& Suarez S. (2008). Soluble adenylyl cyclase is required for activation of sperm but does not have a direct effect on hyperactivation, Reproduction, Fertility and Development; 20(2):247-252.
Mannowetz N , Naidoo NM, Choo S-A, Smith JF ,Lishko PV. (2013).Slo1 is the principal potassium channel of human spermatozoa, eLife; 2: e01009.

Michaut M., Tomes C. N., Blas G. de, Yunes R., and Mayorga L. S. (2000). Calcium-triggered acrosomal exocytosis in humanspermatozoa requires the coordinated activation of Rab3A and -ethylmaleimide-sensitive factor.Proceedings of the National Academy of Sciences of the United States of America; 97(18) 9996-10001.

Nasr-Esfahani MH, Tavalaee M, Deemeh MR, Arbabian M, Parrington J. (2010). Can Assessment of Total Acrosin Activity Help Predict Failed or Low Fertilization Rate ICSI for Implementation of Artificial Oocyte Activation?. The Open Andrology Journal; 2:1926.

Neri QV, Leea B, Rosenwaksa Z, Machacab K, Palermoa GD. (2014). Understanding fertilization through intracytoplasmic sperm injection (ICSI).Cell Calcium; 55: 24-37.

Pietrobon D, Di Virgilio F, Pozzan T. (1990). Structural and functional aspects of calcium homeostasis in eukaryotic cells. Eur. J. Biochem; 193: 599- 622.

Rahman Md S., Kwon W-S., and Pang M-G. , (2014)..Calcium Influx and Male Fertility in the Context of the Sperm Proteome: An Update,. BioMed Research International; 2014:13.

Reed PW \& Lardy HA (1972). A23187: A divalent cation ionophore. J Biol Chem.; 247(21):6970-6977.

Ren D \& Xia J. (2010). Calcium signaling through CatSper channels in mammalian fertilization. Physiology; 25(3):165-175.

Tasripoo K, Srisakwattana K, Nualchuen $\mathrm{W}$ and Sophon S. (2012). Effects of Various Activators on Bovine Embryonic Development Following Intracytoplasmic Sperm Injection. Iranian Journal of Applied Animal Science; 2(2): 167-173.

Tateno H, Krapf D, Hino T, Sánchez-Cárdenas C, Darszon A, Yanagimachi R, Visconti PE. (2013). $\mathrm{Ca}^{2+}$ ionophore A23187 can make mouse spermatozoa capable of fertilizing in vitro without activation of cAMPdependent phosphorylation pathways. Proceedings of the National Academy of Sciences of the United States of America; 110(46): 18543-18548.

Vanden Meerschaut F, Leybaert L, Nikiforaki D, Qian C, Heindryckx B, De Sutter P. (2013). Diagnostic and prognostic value of calcium oscillatory pattern analysis for patients with ICSI fertilization failure. Human Reproduction; 28(1): 87-98. 
Wang X, Sada K, Yanagi S, Yang C, Rezaul K, Yamamura H. (1994). Intracellular calcium dependent activation of p72syk in platelets. J Biochem. ; 116(4):858861.

Zhang J, Ding X, Bian Z, Xia Y, Lu C, Wang S, Song L, Wang X.(2010). The effect of anti-eppin antibodies on ionophore A23187-induced calcium influx and acrosome reaction of human spermatozoa. Human Reproduction;25 (1): 29-36. 